\title{
Novel Hybrid Spectrum Handoff for Cognitive Radio Networks
}

\author{
Nisar A. Lala ${ }^{\mathrm{a}^{*}}$, Moin Uddin ${ }^{\mathrm{b}}, \mathrm{N}$ A Sheikh ${ }^{\mathrm{c}}$ \\ ${ }^{a}$ Division of Agricultural Engineering, SKUAST(K), Srinagar, $J$ \& K, India \\ ${ }^{b}$ Delhi Technological University, Delhi, India \\ ${ }^{c}$ Department of Mathematics, NIT Srinagar, J \& K, India
}

\begin{abstract}
Cognitive radio (CR) is projected as a technology (or solution) that will raise the spectrum utilization considerably by allowing low-priority or secondary user (SU) to utilize the spectrum of high-priority or primary user (PU) opportunistically. Spectrum handoff is a different type of handoff necessitated by the reappearance of the primary user on the frequency channels occupied by the secondary user at that time and location. In this paper, a hybrid type of spectrum handoff algorith $\mathrm{m}$ is proposed where proactive decision and reactive decision approaches are combined. Depending on the arrival rate of primary user (i.e. PU activity), the algorith $\mathrm{m}$ switches from reactive decision mode to proactive decision mode and vice versa. The switching from one mode to another mode depends on threshold value of PU activity and we evaluated the threshold value through analysis for switching of the algorithm to be 0.37 . Simulated results show that the proposed hybrid spectrum handoff algorithm reduces the total service time of secondary user considerably compared to conventional proactive decision or reactive decision handoff approaches.
\end{abstract}

Index Terms: Cognitive radio; spectrum handoff; proactive decision handoff; reactivedecision handoff; queueing theory.

(C) 2013 Published by MECS Publisher. Selection and/or peer review under responsibility of the Research Association of Modern Education and Computer Science

\section{Introduction}

The spectrum is a precious natural resource and is presently regulated by governmental agencies to avoidinterference among users and is allocated by fixed spectrum allocation policy. The fast growth of new wireless applications and services has resulted in increased demand of radio spectrum access. But most of the radio spectrum stands already allocated by fixed allocation policy and it becomes difficult to find unallocated spectrum for these new upcoming applications and services. As per the survey of Federal Communications Commission (FCC) [1], up to $85 \%$ of the assigned spectrum is underutilized. This allocation policy has

* Corresponding author

E-mail address:lalanisar_ae@rediffmail.com 
created a situation where there appears an artificial scarcity of the spectrum. This ever increasing demand of spectrum for new applications cannot be fulfilled unless an alternate scheme to regulate the scarce spectrum is not found. Therefore, FCC has suggested a new communication paradig $\mathrm{m}$ for accessing the assigned spectrum dynamically [2] known as cognitive radio (CR). CR is a key technology that will make the dynamic spectrum access (DSA) a reality. DSA allows the SU to dynamically adjust its operating parameters (such as transmit power, modulation, operating frequency) in order to adapt to time varying radio environment and utilize the idle spectrum opportunistically [3-6], not used by the primary user at that time and location.In addition to spectrum sensing and management, another important function a CR should perform is spectrum mobility and gives rise to a different type of handoff in cognitive radio networks known as spectrum handoff.The aim of spectrum handoff is to help the SU to release the occupied channels instantlyin order to avoid interference to the primary user and find suitable idle channels to restart the interrupted transmission. The handoff to new channels should be seamless so that an application running on the SU perceives minimum quality of service (QoS) degradation [7]. The CR technology allows SUs to sense the environment to find spectrumholes (or idle channel) and utilize these spectrum holes for transmission opportunity with the constraint of noninterference to the primary user. In cognitive radio, the PU has the priority to access the spectrum.

Depending on the decision method used for selecting the idle channels for future handoffs, the spectrum handoff process is classified as the proactive decision and reactive decision handoff approaches.

- In case of proactive decision handoff [8-12], the channels to be used for future handoffs are decided before actual data transmission takes place. The SU senses the wideband spectrum periodically for detection of idle channels so that expected usage pattern of the wideband spectrum over longer period is generated. Then the CR predicts the channels having highest probability of appearing idle at the time of actual handoff.

- In case of the reactive decision handoff [13-14], idle channels are detected through instantaneous sensing of the wideband spectrum after arrival of the PU.

There have been many studies applying queueing theory to study spectrum handoff in cognitive radio networks. Theauthors of [15], proposed the comparative analysis of two approaches namely proactive decision and reactive decision. The analys is of total service time of proactive decision handoff algorith $m$ with multiple interruptions was proposed in [16] while analysis of extended data delivery time of reactive decision was proposed in [17]. The authors of [18] proposed spectrum management techniques in cognitive radio networks with main focus on QoS provisioning.

In this paper, we focus on the performance analysis of the proposed hybrid spectrum handoff algorithm against the conventional proactive decision or reactive decision handoff approaches. The total service time of the algorithm with multiple interruptions is evaluated using pre-emptive resume priority $\mathrm{M} / \mathrm{G} / 1$ queueing network model.

The rest of the paper is organized as follows. Section 2 introduces a PRP M/G/1 queueing network model used to evaluate total service time. Section 3 presents proactive decision spectrum handoff. Section 4 presents reactive decision spectrum handoff. Section 5 proposes the hybrid spectrum handoff algorithm. Section 6 presents the simulated results of the hybrid spectrum handoff algorithm and the conclusion is provided in section 7.

\section{PRP M/G/1 Queueing Network}

A PRP M/G/1 queueing network model [19-25] is used for the characterization of the spectrum usage interactions between primary users and secondary users. The transmission of the SU can be interrupted multip le times by the arrival of PU. Therefore, this model is used to calculate the total service time of the two 


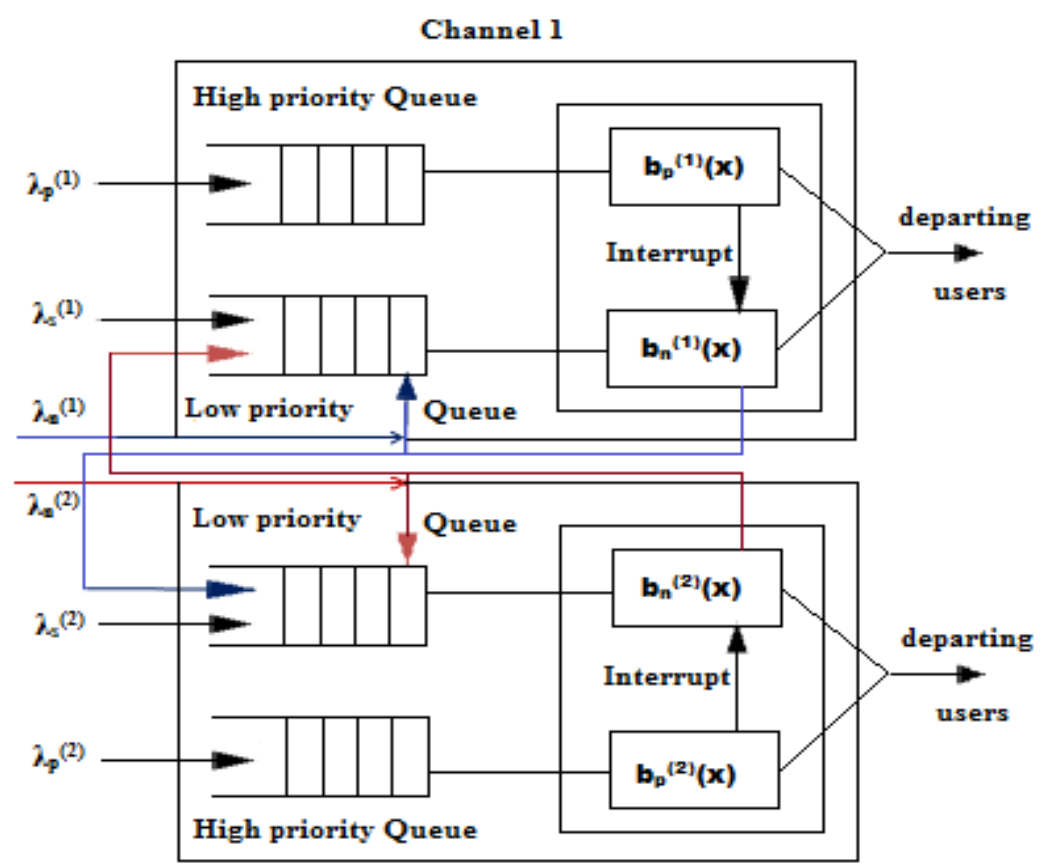

Channel 2

Fig. 1. The PRP M/G/1 queueing net work for two channel system where n ～(Repploduced from [15])

approaches namely proactive decision and reactive decision.Important featuresof PRP M/G/1 queueing network model are given below:

- The transmissions of secondary users have low priority; therefore, can be interrupted by the arrival of PUs.

- When PU arrives back on the channels presently occupied by SU, the transmission of the SU is paused instantly in order to avoid interference.

- The interruptedSU has a choice to transmit either on the same channels or on other idle channels.

- In case of many secondary users contending for channel access, the access to channels is allowed as on first come first served basis.

Fig.1 shows an example of the PRP M/G/1 queueing network with two channels.The PUs are put into the high priority queue while theSUs are put into the low priority queue. The interrupted SU has two options, either to stay on the current channels or change the operating channels. In case of staying on the same channels, the unfinished transmission is put into the head of low-priority queue while in case of changing the operating channels, the unfinished transmission is put into the tail of low-priority queue. In both cases, the interrupted transmission is resumed as soon as the channels become idle. The parameter of importance in this model is the effective packet length and is defined as the duration of the transmission of the packet until PU arrives back on those channels.

\section{Proactive Decision S pectrum Handoff}

In case of proactive decision handoff, the decision for channel switching is taken prior to actual handoff. When PU arrives back, the SU pause its transmission and quickly handoff to the predetermined target channel. In this case, the total service time of SU may be reduced as there is no need of instantaneous spectrum sensing 
and handshaking between the transmitting and receiving SUs. But at the time of actual handoff, the predetermined channels may be busy. Therefore, the SU has to wait in the queue till all the PUs and SUs complete their service. As a consequence of this, there could be an increase in actual handoff delay.

Let $\lambda p$ (arrivals/slot), $\lambda \mathrm{s}$ (arrivals/slot) be the initial arrival rates of the primary users' and secondary users' connection at each channel and Xp (slots/arrival) and Xs (slots/arrival) be their corresponding service time. It is assumed that Xp and Xs are exponentially distributed with service rates $\mu \mathrm{p}$ and $\mu \mathrm{s}$. In this paper, we have decided to change the channels when spectrum handoff takes place. Ifpredetermined channel is idle then the transmission of secondary user is restarted on this new channel and the handoff delay happens to be the switching delay. If the predetermined channel happens to be busy then secondary user has to wait till all primary users and the secondary users in the queue complete their transmission. The handoff delay in this case is switching delay and waiting time on that channel.

Let Tproactive be the average total service time, ts be channel switching time and Ws be waiting time of secondary user on another channel until all primary users and other secondary users finish their transmission. The closed form expression for proactive decision spectrum handoff is derived in [16], we have

$T_{\text {proactive }}=E\left[X_{s}\right]+E[N]\left(W_{s}+t_{s}\right)$

where $\mathrm{E}[\mathrm{N}]$ is the average number of interruptions, $\mathrm{E}\left[\mathrm{X}_{\mathrm{s}}\right]$ is the average secondary service time.

The average number of interruptions for a secondary user within a period of $E\left[\mathrm{X}_{\mathrm{s}}\right]$ can be obtained as

$E[N]=\lambda_{p} E\left[X_{s}\right]$

since the transmission of the secondary users will depend on the primary arrival rate.

The total service time of the proactive handoff scheme comes out as

$T_{\text {proactive }}=E\left[X_{s}\right]+\left(\lambda_{p} E\left[X_{S}\right]\left(\frac{\lambda_{p}\left(E\left[X_{p}\right]\right)^{2}+\frac{\lambda_{s}}{\left(\lambda_{p}+\mu_{s}\right) \mu_{s}}+\frac{\rho_{p}^{2}}{1-\rho_{p}} E\left[X_{p}\right]}{1-\rho_{p}-\rho_{s}}+t_{s}\right)\right.$

where $t_{s}$ is the channel switching time, $E\left[X_{p}\right]$ is the average primary service time, $\rho_{p}$ is primary utilization is equal to $\rho_{\mathrm{p}}=\lambda_{\mathrm{p}} \mathrm{E}\left[\mathrm{X}_{\mathrm{p}}\right]$ and $\rho_{\mathrm{s}}$ is secondary utilization is equal to $\rho_{\mathrm{s}}=\lambda_{\mathrm{s}} \mathrm{E}\left[\mathrm{X}_{\mathrm{s}}\right]$.

\section{Reactive Decision S pectrum Handoff}

In case of reactive decision handoff, idle channels are found through instantaneous sensing of the wideband spectrum at the time of the actual handoff. The unfinished transmission of the interrupted SU will be restarted on these idle channels. In this approach, the handoff delay may be short as the target idle channels are found reliably through instantaneous sensing of the spectrum and the handoff delay is the sensing time plus the handshaking time necessary for consensus of the target idle channels between the transmitting and receiving SUs. Hence, time duration of spectrum sensing and handshaking is an important factor for assessing the performance of this approach.

The closed form expression is derived in [17] for total service time for reactive decision spectrum handoff as

$T_{\text {reactive }}=E\left[X_{\mathrm{s}}\right]+E[D]$

where $\mathrm{E}\left[\mathrm{X}_{\mathrm{s}}\right]$ is the average service time of secondary users and $\mathrm{E}[\mathrm{D}]$ is the average cumulative handoff delay. Then total service of reactive decision handoff $\left(\mathrm{T}_{\text {reactive }}\right)$ comes out as 
$T_{\text {reactive }}=E\left[X_{S}\right]+\frac{\lambda_{p}\left[t_{p} \mu_{s}+\left(E\left[X_{p}\right]\right)^{2} \lambda_{p} \mu_{S}+E\left[X_{p}\right]\left(\lambda_{s}-t_{p} \lambda_{p} \mu_{S}\right)\right]}{\left(1-\lambda_{p} E\left[X_{p}\right]\right)\left(\mu_{S}\right)^{2}}$

where $t_{p}$ is the processing time and is given as $t_{p}=t_{s}+t_{f}, t_{s}$ is channel switching time, $t_{f}$ channel sensing time and $\mu_{\mathrm{s}}$ is the secondary service rate equal to $\mu_{\mathrm{s}}=1 / \mathrm{E}\left[\mathrm{X}_{\mathrm{s}}\right]$ and $\mu_{\mathrm{p}}$ is the primary service rate equal to $\mu_{\mathrm{p}}=$ $1 / \mathrm{E}\left[\mathrm{X}_{\mathrm{p}}\right]$.

\section{Proposed Hybrid Spectrum Handoff Algorithm}

A hybrid type of spectrum handoff is proposed in this section,which is the combination of proactive decision and reactive decision approaches. Depending on the PUactivity, the algorithm switches from proactive decision mode to reactive decision mode and vice versa. Equation (3) determines total service time of proactive decision (i.e. Tproactive) approach, while equation (5) determines total service time of reactive decision (Treactive) approach. As can be seen from equations (3) and (5), the total service time of both decision approaches depend on average secondary service rate ( $\mu \mathrm{s})$, average primary service rate ( $\mu$ ), secondary arrival rate $(\lambda s)$ and mean sensing time (tp).For different arrival rates of PU, the total service time of both decision approaches change due to change in number of perceived handoffs. In order to simplify the analysis of the proposed algorithm, it is assumed that each channel has identical traffic patterns and the hybrid handoff algorithm is simulated for two channel scenario. It is also assumed that channel switching time (ts) is equal to zero so that channel processing time (tp) is equal to the sensing time only.

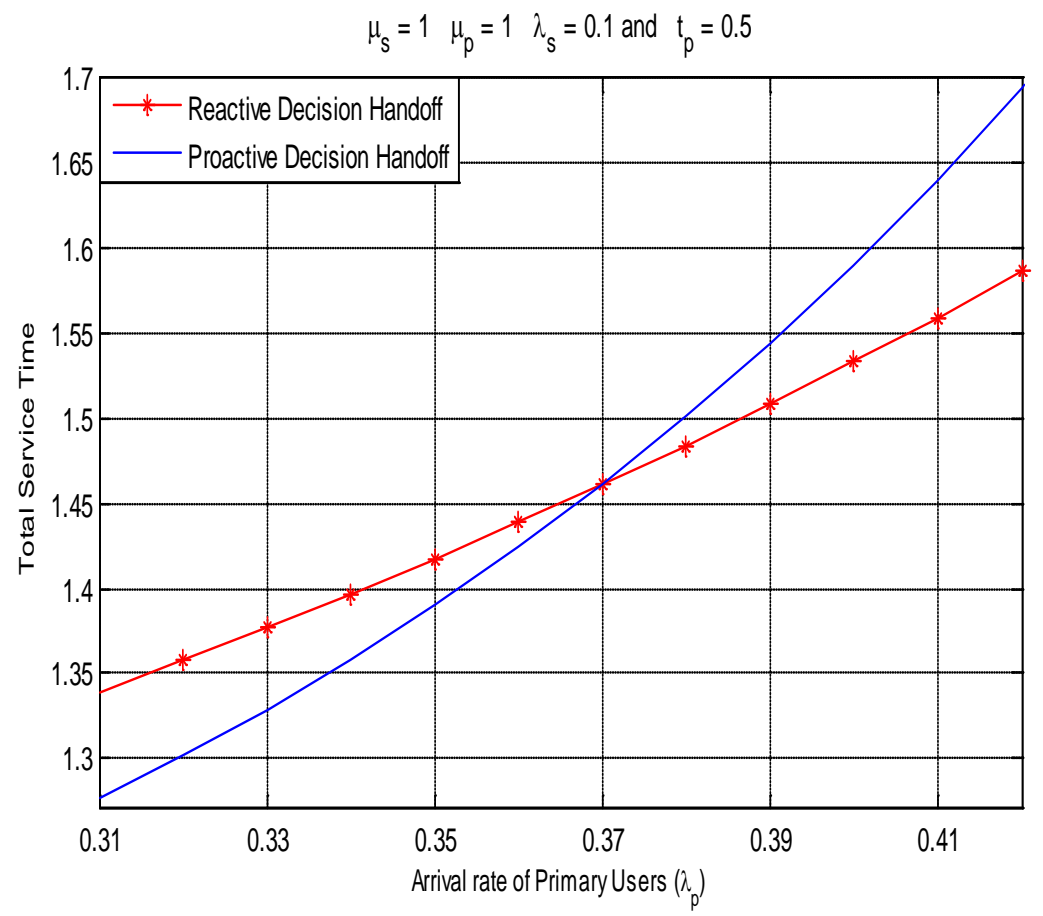

Fig.2. Comparison of reactive decisionand proactive decisionhandoff approaches for threshold evaluation 

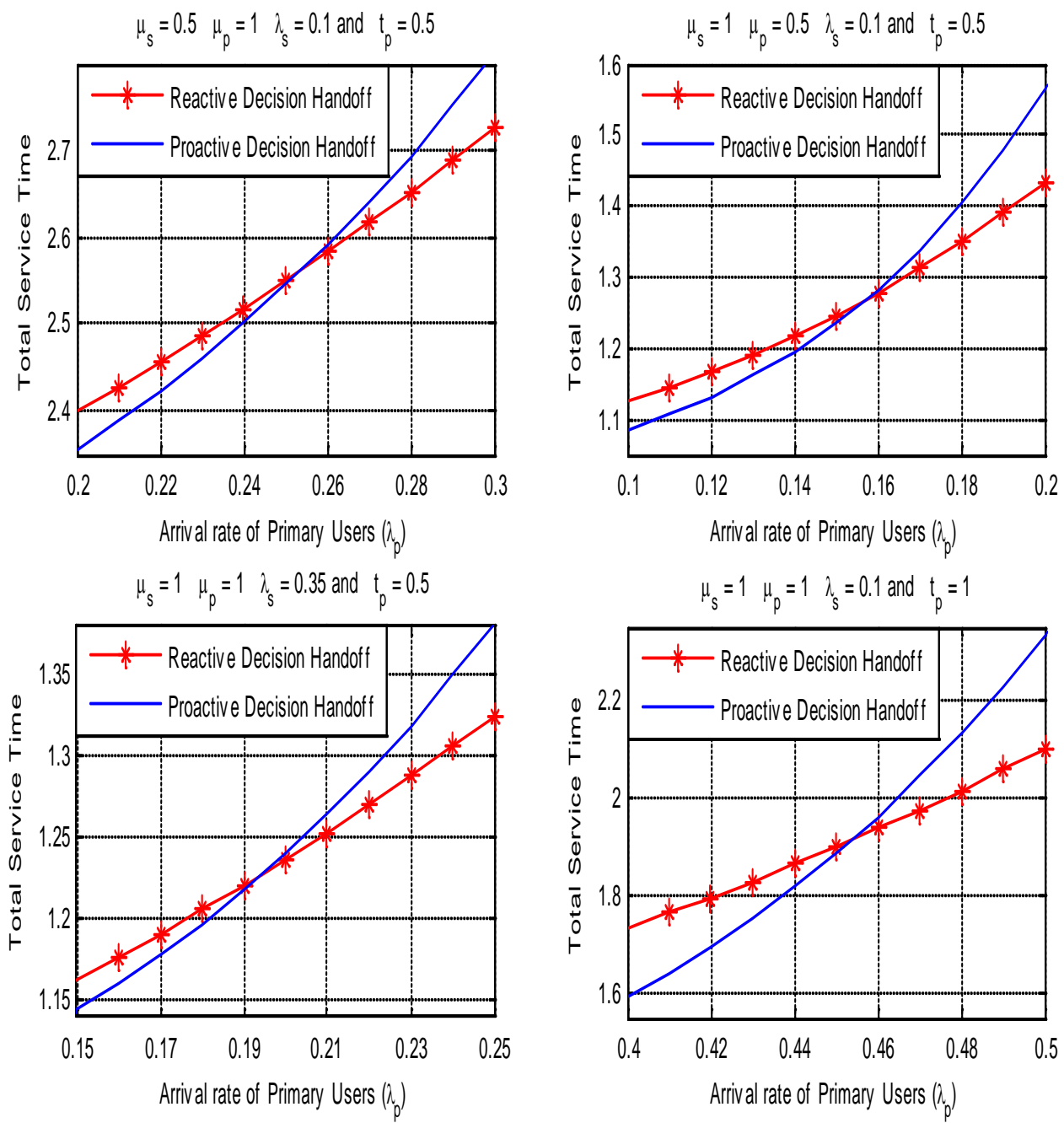

Fig.3. Sensitivity of $\mu s, \mu p, \lambda s$ and tp parameters on threshold value of $\lambda p$

\section{Simulated Results}

In this section, we presented the simulated results of the proposed hybrid handoff algorithm to support our analysis and the analysis of the algorithm is performed in Matlab 7.6. The first step for the analys is is to determine the value of threshold to be used for switching between proactive decision and reactive decision approaches and vice versa. In Fig. 2, we plot the total service time of proactive decision and reactive decision approaches as a function of primary arrival rate $(\lambda p)$. As can be seen from Fig. 2, for parameter values such as $\mu \mathrm{s}=1, \mu \mathrm{p}=1, \lambda \mathrm{s}=0.1$ and $\mathrm{t}=0.5$, the threshold value of primary arrival rate $(\lambda \mathrm{p})$ comes out to be 0.37 . We have analysed, the sensitivity of threshold value to these parameters, such as average secondary service rate ( $\mu \mathrm{s})$, 


$$
\mu_{\mathrm{s}}=1 \quad \mu_{\mathrm{p}}=1 \quad \lambda_{\mathrm{s}}=0.1 \text { and } \mathrm{t}_{\mathrm{p}}=0.5
$$

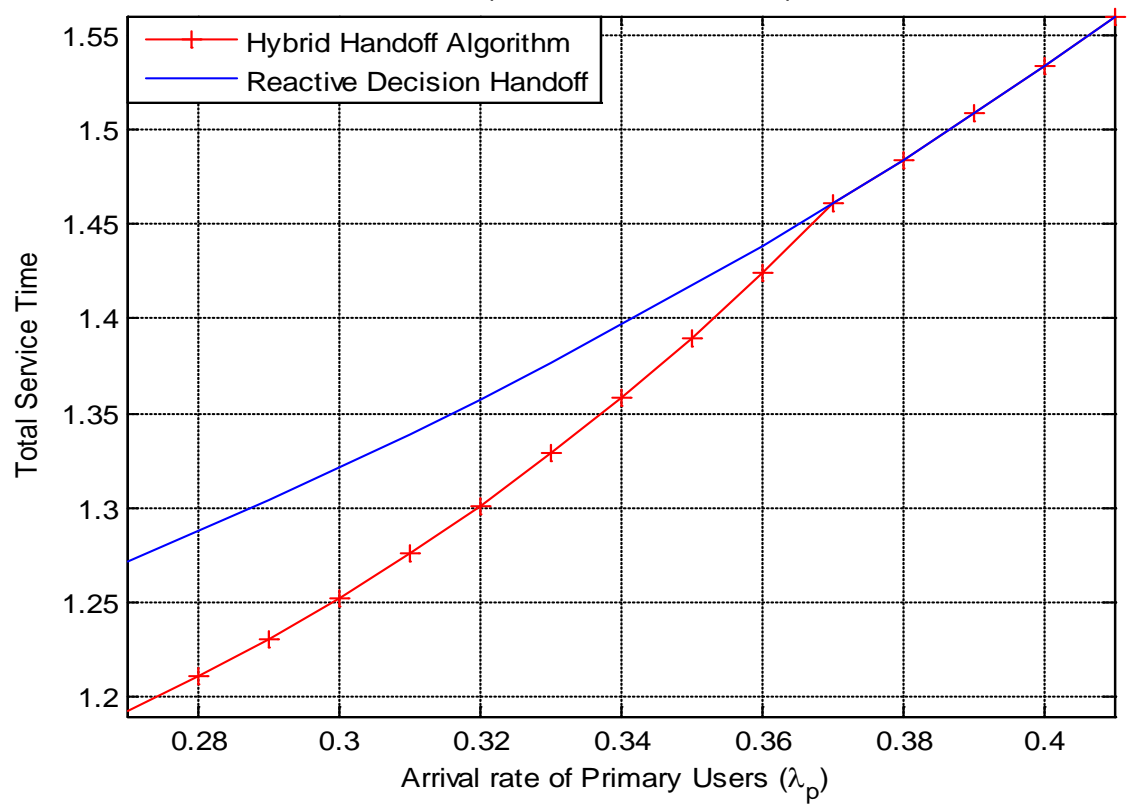

Fig. 4. Comparison of total service time of hybrid handoff and reactive decision handoffalgorithms

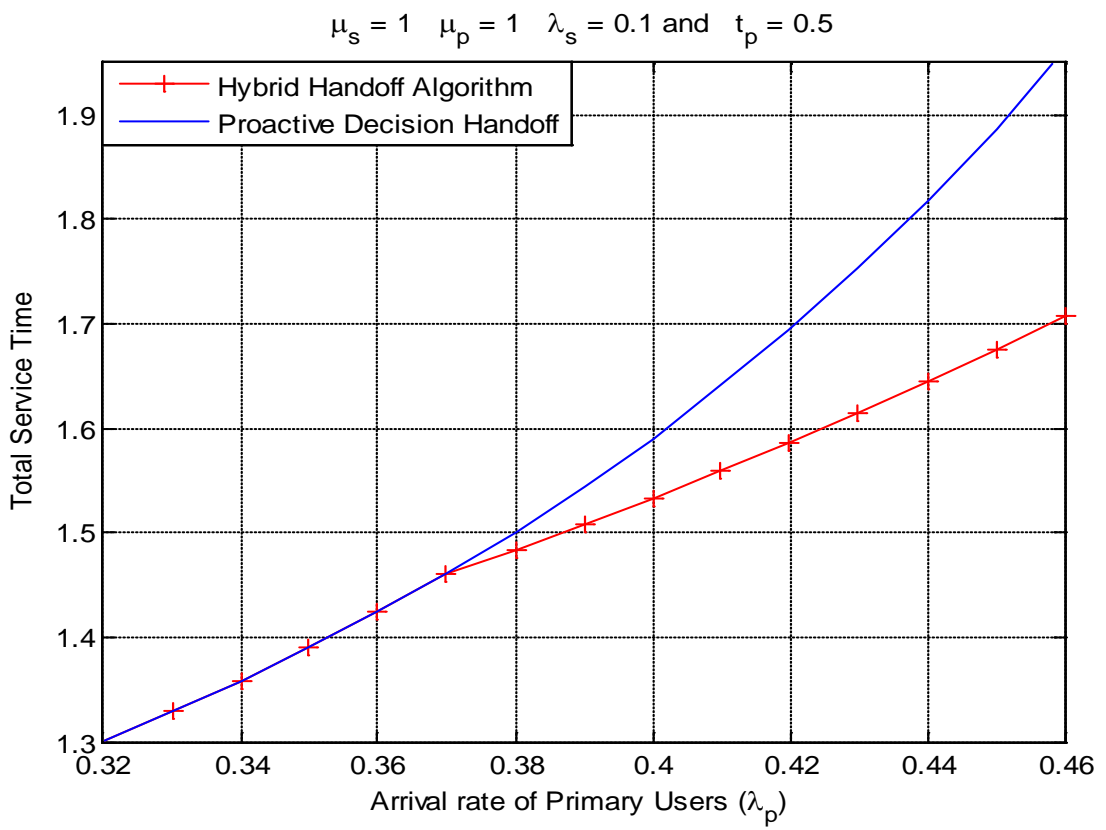

Fig. 5. Comparison of total servicetime of hybrid handoff and proactive decision handoffalgorithms 
average primary service rate $\left(\mu_{\mathrm{p}}\right)$, secondary arrival rate $\left(\lambda_{\mathrm{s}}\right)$ and mean sensing time $\left(\mathrm{t}_{\mathrm{p}}\right)$ with reference values of parameters such as $\mu_{s}=1, \mu_{p}=1, \lambda_{s}=0.1$ and $t_{p}=0.5$. Fig. 3 presents the impact analysis of varying one parameter at a time to threshold value in comparison to reference values. As can be seen from Fig. 3, for parameter values of $\mu_{s}=0.5, \mu_{p}=1, \lambda_{s}=0.1$ and $t_{p}=0.5$, the threshold value $\lambda_{p}=0.252$, for parameter values $\mu_{s}=1$, $\mu_{p}=0.5, \lambda_{s}=0.1$ and $t_{p}=0.5$, the threshold value $\lambda_{p}=0.155$, for parameter values of $\mu_{s}=1, \mu_{p}=1, \lambda_{s}=0.35$ and $\mathrm{t}_{\mathrm{p}}=0.5$, the threshold value $\lambda_{\mathrm{p}}=0.192$ and for parameter values of $\mu_{\mathrm{s}}=1, \mu_{\mathrm{p}}=1, \lambda_{\mathrm{s}}=0.1$ and $\mathrm{t}_{\mathrm{p}}=1$, the threshold value $\lambda_{\mathrm{p}}=0.453$.

Fig. 4 presents the comparis on of the proposed hybrid algorithm with reactive decision handoff approach. As can be seen from Fig. 4 , the service time of the hybrid algorithm is lower until $\lambda p<0.37$. For $\lambda p \geq 0.37$, the service time of both the algorithms is same. At lower values of $\lambda p$ (i.e. $\lambda p<0.37$ ), there is highest probability that the predetermined channels will appear to be idle at the time of actual handoff. In this case, handoff delay is only the switching delay and our hybrid algorithm experiences lesser number of handoffs which results in reduction of total service time of the SU.

Fig. 5 presents the comparison between the proposed hybrid algorithm with proactive decision handoff algorithm. As can be seen from Fig. 5, the service time of the of both algorithms is equal until $\lambda p \leq 0.37$. For $\lambda p>0.37$, the service time of the hybrid algorithm is lower than the proactive decision algorithm. At higher values of $\lambda$ p (i.e. $\lambda p>0.37$ ), there is highest probability that the predetermined channels appear busy at the time of actual handoff. Therefore, SU will spend most of the time waiting in queue for service while reactive decision handoff approach will find idle channels reliably by instantaneous sensing. The total delay in this case is sensing and switching delay which is considerably lower than the waiting time in a queue.

Therefore, the proposed hybrid handoff algorithm will intelligently operate in proactive decision handoff mode when $\lambda p^{<} 0.37$, will switch to reactive decision handoff mode when $\lambda p=0.37$ and remains in this mode until $\lambda p>0.37$. The results demonstrate that there is considerable reduction in total service time of the secondary users.

\section{Conclusion}

We have proposed and analysed a hybrid spectrum handoff algorith m for total service time of the secondary user. The proposed hybrid algorithm switches from proactive decision mode to reactive decision mode and vice versa, depending on the threshold value of the primary arrival rate. We have analyzed the sensitivity of selected parameters such as $\mu s, \mu p, \lambda s$ and tp on threshold value used for switching of the hybrid algorithm. The resultsdemonstrate that for the parameter values $\mu \mathrm{s}=1, \mu \mathrm{p}=1, \lambda \mathrm{s}=0.1$, and tp $=0.5$, the threshold value of arrival rate of primary users $(\lambda p)$ comes out to be $\lambda p=0.37$. The simulated results show that the proposed hybrid handoff algorithm reduces the total service time of SUs considerably as compared to conventional proactive decision or reactive decision handoff algorithms. The reduction in total service time results in increased throughput and will support higher quality of service (QoS) for SUs.

\section{References}

[1] FCC.Notice of proposed rulemaking and order. No. 03-222, Dec. 2003.

[2] Et docket No. 03-237, Nov. 2003.[Online]Available:http://hraunfoss.fcc.gov/edocs public/attachmatch/FCC-03- 289A1.pdf

[3] Mitola J. "Cognitive radio: an integrated agentarchitecture for software defined radio".Ph.D. Dissertation:KTH Royal Institute of Technology; 2000.

[4] Mitola J,Maguire GQ. “Cognitive radio: makingsoftware radios morepersonal”.IEEE Personal Communications 1999; 6(4):13-8.

[5] Haykin S. "Cognitive radio: Brain empowered wireless communications". IEEEJournalon Selected Areas in Communications 2005; 23(2): 201-20. 
[6] Akyildiz IF, LeeWY, VuranMC, MohantyS.“Next generation/dynamic spectrum access/cognitive radiowireless networks: A survey”.Computer Networks (Elsevier) 2006; 50:2127-59.

[7] Liu HJ, WangZX, LlSF, Yl M. "Study on the performance of spectrum mobility in cognitive wireless Network”. In Proceedings of (IEEE) InternationalConference on Commun ication Systems (ICCS) 2008.

[8] SongY, XieJ. "Proactive spectrum handoff incognitive radioadhoc networks based on commonhoppingcoordination”.In Proceedings of (IEEE) INFOCOM 2010.

[9] Zheng S, YangX, ChenS, LouC. "Target channelsequence selection scheme for proactivedecisionspectrum handoff”. IEEE co mmun ications letters 2011; 15(12):1332-4.

[10] SongY, Xie J."ProSpect:A proactive spectrum handoff framework for cognitive radio adhoc networks withoutcommoncontrolchannel”.IEEE Transactions on mobile computing2012; 11(7):1127-39.

[11] SrinivasaS, JafarSA. "Thethroughput potential of cognitive radio: Atheoreticalperspective”. IEEECommunications Magazine 2007; 73-9.

[12] ShiQ, TaubenheimD, KyperountasS, Gorday P, CorrealN. "Link maintenanceprotocol for cognitiveradiosystem withOFDM PHY”.IEEE InternationalSymposium on Dynamic Spectrum Access Networks (DySPAN) 2007.

[13] [13] Willkomm D, Gross J, Wolisz A. “Reliablelinkmaintenance incognitiveradiosystems”. IEEEInternational Sy mposium on DynamicSpectrum Access Networks (DySPA N) 2005.

[14] TianJ, BiG. "Anewlink maintenance andcompensationmodel forcognitive UWBradio systems". In Proceedings ofInternational Conference on ITS Telecommunications 2006.

[15] Wang L-C, Wang C-W."Spectrum handoff for cognitive radio networks: reactive sensing orproactivesensing?”.IEEE International Performance Computing and Communications Conference (IPCCC) 2008.

[16] Wang C-W, Wang L-C. "Modeling and analysis for proactive decision spectrum handoff in cognitive radio networks”. IEEE International Conference on Commun ications (ICC) 2009.

[17] Wang C-W, WangL-C, Adachi F. "Modeling and analysis for reactive decision spectrum handoff in cognitive radio networks”. IEEE GLOBECOM2010.

[18] Wang L-C, Wang C-W. "Spectrum management techniques with QoS provisioning in cognitive radio networks”. IEEE International Sy mposium on Wireless Pervasive Co mputing 2010.

[19] Zhang C, Wang X, Li J. “Cooperativecognitiveradio withprio rity queueinganalysis”. IEEE International Conference on Communications (ICC) 2009.

[20] SulimanI, Lehtomaki J. "Queueing analysis of opportunisticaccess in cognitiveradios". IEEEInternational Workshop on Cognitive Radio and Advanced Spectrum Management (CogART) 2009.

[21] $\mathrm{Li} \quad \mathrm{H}$. "Queuing analy sisofdy namicspectrumaccesssubjecttointerruptions fromprimary users”.InternationalConferenceonCognitiveRadio OrientedWireless Networks andCo mmunications(Crown Com) 2010.

[22] Shiang H-P,Van der Schaar M. "Queuingbaseddynamic channelselection forheterogeneous multimed iaapplicationsovercognitiveradionetworks”. IEEE Transactions on Multimedia 2008; 10(5):896-909.

[23] BoseSK. “An Introduction toqueueing systems”. Kluwer Academic/Plenum Publishers, New York; 2002.

[24] Wang L-C, Wang C-W, Feng K-T. "A queueing theoretical framework forQoSenhanced spectrummanagement in cognitive radio networks”.IEEE Wireless Commun ications 2011:18-26.

[25] Cooper RB. “Introduction toqueueing theory”. 2nd ed. Elsevier North HollandInc; 1981. 


\section{Authors' Profiles}

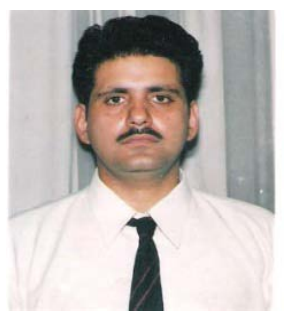

Mr.Nisar Ahmad Lala received his B. Sc. and M.Sc. in Electronics in 1990 and 1994 respectively from University of Kashmir, J\&K, India. He is working as Assistant Professor in the division of Agricultural Engineering, S. K. University of Agricultural Sciences and Technology (Kashmir) Srinagar, J \& K, India. He is currently pursuing Ph.D at National Institute of Technology Srinagar, J\&K, India. His research interests include Cognitive Radio Networks and Fu zzy modelling.

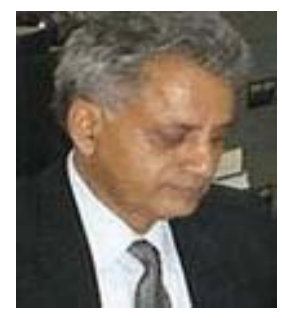

Prof. Moin Uddin is a senior member, IEEE. Moin Uddin did his B.Sc and M.Sc. Engineering in 1972 and1978 from Aligarh Muslim University, Uttar Pardesh, India. He completed his Ph.D in 1993 from Roorkee University, India.He has more than 30 years of experience in academics and research and is currently serving as ProViceChancellor, Delhi Technological University, Delhi. Prior to this, he was Director of Dr. B R A mbedkar National Institute of Technology, Jalandhar, India. He has large number of publications in International and national Journals and fourteen research scholars have completed their Ph.D underhis guidance and five more are pursuing the same. He has designed the computer engineering curriculums of many international and national universities and institutions and is among the expert panel of these universities. Prof. Moin Uddin is a life member ISTE national society and member, board of studies of many institutions.

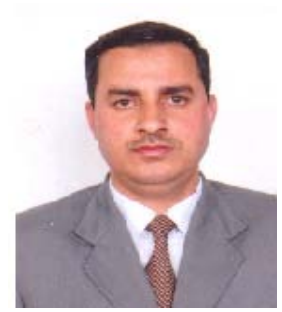

Dr. Neyaz Ahmad Sheikh received his M.Sc, M.phil and Ph.D (Applied Mathematics) in 1991, 1993 and 1996 from Aligarh Muslim University, Uttar Pardesh, India. He is working as Assistant Professor in the department of mathematics, National Institute of Technology Srinagar, J \& K, India. He has fourteen publications in International and national Journals. His research interests include Walsh functions and applications, Functional analysis and some results on wavelets. 